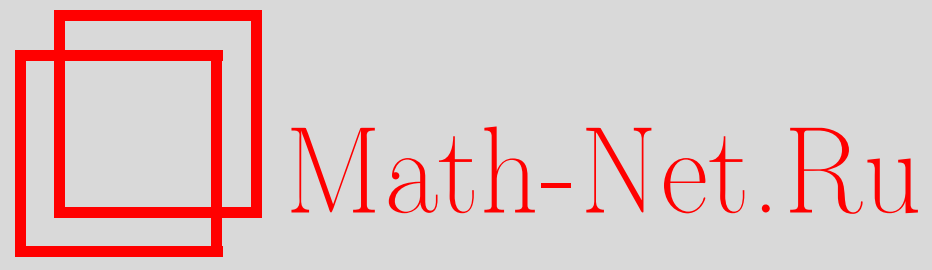

А. А. Андреев, Ю. О. Яковлева, Характеристическая задача для системы гиперболических дифференциальных уравнений третьего порядка общего вида с некратными характеристиками, Вестн. Сам. гос. техн. ун-та. Сер. Физ.-мат. науки, 2013, выпуск 1(), 31-36

DOI: https://doi.org/10.14498/vsgtu1182

Использование Общероссийского математического портала Math-Net.Ru подразумевает, что вы прочитали и согласны с пользовательским соглашением

http://www . mathnet.ru/rus/agreement

Параметры загрузки:

IP : 54.209 .52 .79

26 апреля 2023 г., 12:55:51

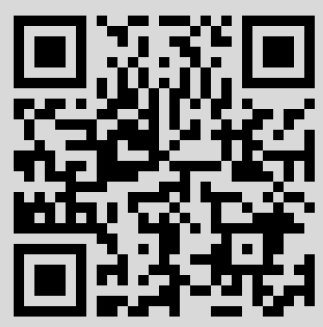


УДК 517.956.3

\title{
ХАРАКТЕРИСТИЧЕСКАЯ ЗАДАЧА ДЛЯ СИСТЕМЫ ГИПЕРБОЛИЧЕСКИХ ДИФФЕРЕНЦИАЛЬНЫХ УРАВНЕНИЙ ТРЕТЬЕГО ПОРЯДКА ОБЩЕГО ВИДА С НЕКРАТНЫМИ ХАРАКТЕРИСТИКАМИ
}

\author{
А.А. Андреев, Ю.О. Яковлева \\ Самарский государственный технический университет, \\ Россия, 443100, Самара, ул. Молодогвардейская, 244. \\ E-mails: andre01071948@yandex.ru, julia.yakovleva@mail.ru
}

\begin{abstract}
Исследуется корректная по Адамару постановка характеристической задачи для системы гиперболических дифференииальных уравнений третъего порядка общего вида с некратными характеристиками. Решение указанной задачи построено в явном виде. Приведён пример аналога задачи Гурса для одной системы гиперболических дифференциальных уравнений третьего порядка.
\end{abstract}

Ключевые слова: система гиперболических дифференциальных уравнений общего вида, некратные характеристики, характеристическая задача, корректность по Адамару.

Исследованию начально-краевых задач для гиперболических уравнений и систем с двумя независимыми переменными порядка выше второго в случае кратных характеристик посвящены работы многих авторов. Изучены граничные задачи относительно корректной постановки их по Ж. Адамару [1-4]. Но характеристические задачи для систем и уравнений гиперболического типа в частных производных с некратными характеристиками исследованы недостаточно. В монографии А. В. Бицадзе [5] приводятся примеры, показывающие, что для системы второго порядка с некратными характеристиками задача Гурса является некорректной по Адамару.

В настоящей работе сформулирована и исследована характеристическая задача для системы гиперболических уравнений третьего порядка общего вида с некратными характеристиками. Установлены достаточные условия её корректности.

1. Предварительные сведения. Рассмотрим систему дифференциальных уравнений в частных производных третьего порядка общего вида с двумя независимыми переменными $x, y \in \mathbb{R}$ на плоскости, не содержащую производных порядка меньше третьего

$$
A^{*} U_{x x x}+B^{*} U_{x x y}+C^{*} U_{x y y}+D^{*} U_{y y y}=0,
$$

где $U(x, y)=\left(u^{1}(x, y), u^{2}(x, y)\right)$ - искомая двумерная вектор-функция, $A^{*}$, $B^{*}, C^{*}, D^{*}$ - постоянные квадратные матрицы второго порядка.

В предположении, что $D^{*}$ - невырожденная матрица, система (1) редуцируется к следующему виду:

$$
A U_{x x x}+B U_{x x y}+C U_{x y y}+U_{y y y}=0 .
$$

Александр Анатолвевич Андреев (к.ф.-м.н., доц.), доцент, каф. прикладной математики и информатики. Юлия Олеговна Яковлева, аспирант, каф. прикладной математики и информатики. 
Пусть матрицы $A, B, C$ попарно коммутативны, тогда без ограничения общности они имеют следующий вид:

$$
\begin{gathered}
A=\left(\begin{array}{ll}
a_{11} & a_{12} \\
a_{21} & a_{22}
\end{array}\right), \quad B=\left(\begin{array}{cc}
b_{22}+b_{12}\left(\frac{a_{11}-a_{22}}{a_{12}}\right) & b_{12} \\
b_{12} \frac{a_{12}}{a_{12}} & b_{22}
\end{array}\right), \\
C=\left(\begin{array}{cc}
c_{11} & c_{21} \frac{a_{12}}{a_{21}} \\
c_{21} & c_{11}-c_{21}\left(\frac{a_{11}-a_{22}}{a_{21}}\right)
\end{array}\right), \\
a_{i j}, b_{i j}, c_{i j} \in \mathbb{R}, \quad a_{i j}, b_{i j}, c_{i j} \neq 0, \quad i, j=1,2 .
\end{gathered}
$$

Матрицы преобразования

$$
\begin{gathered}
T=\left(\begin{array}{ll}
t_{1}\left(b_{22}+\frac{b_{12}}{a_{12}}\left(a_{1}-a_{22}\right)\right) b_{12} \delta_{1}^{-1} & t_{2}\left(b_{22}+\frac{b_{12}}{a_{12}}\left(a_{2}-a_{22}\right)\right) b_{12} \delta_{2}^{-1} \\
t_{1} & t_{2}
\end{array}\right), \\
T^{-1}=\frac{a_{12} \delta_{1} \delta_{2}}{b_{12}^{2} d_{B}\left(a_{2}-a_{1}\right) t_{1} t_{2}}\left(\begin{array}{rr}
t_{2} & -t_{2}\left(b_{22}+\frac{b_{12}}{a_{12}}\left(a_{2}-a_{22}\right)\right) b_{12} \delta_{2}^{-1} \\
-t_{1} & t_{1}\left(b_{22}+\frac{b_{12}}{a_{12}}\left(a_{1}-a_{22}\right)\right) b_{12} \delta_{1}^{-1}
\end{array}\right),
\end{gathered}
$$

где

$$
\begin{gathered}
d_{B}=b_{22}^{2}+b_{12} b_{22}\left(\frac{a_{11}-a_{22}}{a_{12}}\right)-b_{12}^{2} \frac{a_{21}}{a_{12}}, \quad \delta_{1}=\left(b_{22}+\frac{b_{12}}{a_{12}}\left(a_{1}-a_{22}\right)\right) b_{22}-d_{B} \\
\delta_{2}=\left(b_{22}+\frac{b_{12}}{a_{12}}\left(a_{2}-a_{22}\right)\right) b_{22}-d_{B}, \quad t_{1}, t_{2} \in \mathbb{R}, \quad t_{1}, t_{2} \neq 0
\end{gathered}
$$

одновременно приводят матрицы $A, B, C$ к диагональной форме: $T^{-1} A T=$ $=\Lambda_{A}=\operatorname{diag}\left(a_{1}, a_{2}\right), T^{-1} B T=\Lambda_{B}=\operatorname{diag}\left(b_{1}, b_{2}\right), T^{-1} C T=\Lambda_{C}=\operatorname{diag}\left(c_{1}, c_{2}\right)$, при этом $a_{1}, a_{2}, b_{1}, b_{2}, c_{1}, c_{2}$ - различные собственные значения матриц $A, B$, $C$ соответственно.

Система (2) эквивалентна следующей системе:

$$
\Lambda_{A} V_{x x x}+\Lambda_{B} V_{x x y}+\Lambda_{C} V_{x y y}+V_{y y y}=0
$$

или

$$
\left\{\begin{array}{l}
a_{1} v_{x x x}^{1}+b_{1} v_{x x y}^{1}+c_{1} v_{x y y}^{1}+v_{y y y}^{1}=0 \\
a_{2} v_{x x x}^{2}+b_{2} v_{x x y}^{2}+c_{2} v_{x y y}^{2}+v_{y y y}^{2}=0
\end{array}\right.
$$

Каждое характеристическое уравнение этой системы (3) имеет три различных корня: $\lambda_{1}, \lambda_{2}, \lambda_{3}$ и $\mu_{1}, \mu_{2}, \mu_{3}$, соответственно.

2. Характеристическая задача. В работе [6] приводятся пример, иллюстрирующий некорректность по Адамару классической постановки задачи Гурса для гиперболического уравнения третьего порядка с некратными характеристиками, и решение характеристической задачи, корректной по Адамару, для гиперболического уравнения третьего порядка общего вида

$$
a v_{x x x}+b v_{x x y}+c v_{x y y}+v_{y y y}=0
$$

с некратными характеристиками $y-\lambda_{1} x+C_{1}, y-\lambda_{2} x+C_{2}, y-\lambda_{3} x+C_{3}$. 
Пусть $x \in I_{c} \equiv[a, b], c=(a+b) / 2$. Отрезок $I_{c}$ имеет центральную симметрию: $\forall x \in I_{c}, 2 c-x \in I_{c}$, тогда для любой функции $f(x)$ справедливы выражения

$$
f(x)=f_{\mathrm{H}}^{c}+f_{\mathrm{\Psi}}^{c}, \quad f_{\mathrm{H}}^{c}=\frac{1}{2}(f(x)-f(2 c-x)), \quad f_{\mathrm{\Psi}}^{c}=\frac{1}{2}(f(x)+f(2 c-x)) .
$$

Функции $f_{\mathrm{H}}^{c}, f_{\mathrm{ч}}^{c}$ при $c=0$ будем обозначать $f_{\mathrm{H}}, f_{\mathrm{ч}}$ соответственно.

Для системы (2) рассмотрим следующую характеристическую задачу.

ЗАДАчА $\mathrm{G}$. Найти решение $U(x, y) \in C^{3}(\mathbb{R} \times \mathbb{R})$ системы уравнений $(2)$, удовлетворяющее условиям

$$
\begin{array}{ll}
\left\langle l_{1}, U\left(x, \lambda_{1} x\right)\right\rangle=\alpha^{1}(x), & \left\langle l_{2}, U\left(x, \mu_{1} x\right)\right\rangle=\alpha^{2}(x), \\
\left\langle l_{1}, U\left(x, \lambda_{2} x\right)\right\rangle=\beta^{1}(x), & \left\langle l_{2}, U\left(x, \mu_{2} x\right)\right\rangle=\beta^{2}(x) \\
\left\langle l_{1}, U\left(x, \lambda_{3} x\right)\right\rangle=\gamma^{1}(x), & \left\langle l_{2}, U\left(x, \mu_{3} x\right)\right\rangle=\gamma^{2}(x)
\end{array}
$$

где $\alpha^{i}(x), \beta^{i}(y), \gamma^{i}(x) \in C^{3}(\mathbb{R}), i=1,2,\langle a, b\rangle$ - скалярное произведение;

$$
\begin{aligned}
& l_{1}=\left(\frac{a_{12} \delta_{1} \delta_{2}}{b_{12}^{2} d_{B}\left(a_{2}-a_{1}\right) t_{1}},-\frac{a_{12}\left(b_{22}+\frac{b_{12}}{a_{12}}\left(a_{2}-a_{22}\right)\right) \delta_{1}}{b_{12} d_{B}\left(a_{2}-a_{1}\right) t_{1}}\right), \\
& l_{2}=\left(-\frac{a_{12} \delta_{1} \delta_{2}}{b_{12}^{2} d_{B}\left(a_{2}-a_{1}\right) t_{2}}, \frac{a_{12}\left(b_{22}+\frac{b_{12}}{a_{12}}\left(a_{1}-a_{22}\right)\right) \delta_{1}}{b_{12} d_{B}\left(a_{2}-a_{1}\right) t_{2}}\right) .
\end{aligned}
$$

Tеорема 1. Ecлu $\gamma_{\mathrm{H}}^{i}(x)=\alpha_{\mathrm{H}}^{i}\left(\sigma_{i} x\right)+\beta_{\mathrm{H}}^{i}\left(\left(1-\sigma_{i}\right) x\right), i=1,2$, əде

$$
\sigma_{1}=\left(\lambda_{3}-\lambda_{2}\right) /\left(\lambda_{1}-\lambda_{2}\right), \quad \sigma_{2}=\left(\mu_{3}-\mu_{2}\right) /\left(\mu_{1}-\mu_{2}\right),
$$

то задача $G$ корректна по Адамару.

До каз а те ль ст в о. Система (2) преобразованием $T$ редуцируется к системе (3). Решение каждого уравнения этой системы [6] имеет следующий вид:

$$
\begin{aligned}
& v^{1}(x, y)=\alpha^{1}\left(\frac{y-\lambda_{2} x}{\lambda_{1}-\lambda_{2}}\right)+\beta^{1}\left(\frac{y-\lambda_{1} x}{\lambda_{2}-\lambda_{1}}\right)-\frac{1}{2} \alpha^{1}(0)+ \\
& +\frac{1}{2}\left[\alpha_{\mathrm{ч}}^{1}\left(\frac{y-\lambda_{3} x}{\lambda_{1}-\lambda_{3}}\right)-\alpha_{\mathrm{u}}^{1}\left(\frac{y-\lambda_{2} x}{\lambda_{1}-\lambda_{2}}\right)-\alpha_{\mathrm{u}}^{1}\left(\frac{\left(y-\lambda_{1} x\right)\left(\lambda_{2}-\lambda_{3}\right)}{\left(\lambda_{1}-\lambda_{3}\right)\left(\lambda_{1}-\lambda_{2}\right)}\right)\right]+ \\
& +\frac{1}{2}\left[\beta_{\mathrm{ч}}^{1}\left(\frac{y-\lambda_{3} x}{\lambda_{2}-\lambda_{3}}\right)-\beta_{\mathrm{ч}}^{1}\left(\frac{y-\lambda_{1} x}{\lambda_{1}-\lambda_{2}}\right)-\beta_{\mathrm{ч}}^{1}\left(\frac{\left(y-\lambda_{2} x\right)\left(\lambda_{1}-\lambda_{3}\right)}{\left(\lambda_{1}-\lambda_{2}\right)\left(\lambda_{2}-\lambda_{3}\right)}\right)\right]- \\
& -\frac{1}{2}\left[\gamma_{\mathrm{ч}}^{1}\left(\frac{\left(y-\lambda_{3} x\right)\left(\lambda_{1}-\lambda_{2}\right)}{\left(\lambda_{1}-\lambda_{3}\right)\left(\lambda_{2}-\lambda_{3}\right)}\right)-\gamma_{\mathrm{ч}}^{1}\left(\frac{y-\lambda_{1} x}{\lambda_{1}-\lambda_{3}}\right)-\gamma_{\mathrm{ч}}^{1}\left(\frac{y-\lambda_{2} x}{\lambda_{2}-\lambda_{3}}\right)\right], \\
& v^{2}(x, y)=\alpha^{2}\left(\frac{y-\mu_{2} x}{\mu_{1}-\mu_{2}}\right)+\beta^{2}\left(\frac{y-\mu_{1} x}{\mu_{2}-\mu_{1}}\right)-\frac{1}{2} \alpha^{2}(0)+ \\
& +\frac{1}{2}\left[\alpha_{\mathrm{ч}}^{2}\left(\frac{y-\mu_{3} x}{\mu_{1}-\mu_{3}}\right)-\alpha_{\mathrm{ч}}^{2}\left(\frac{y-\mu_{2} x}{\mu_{1}-\mu_{2}}\right)-\alpha_{\mathrm{ч}}^{2}\left(\frac{\left(y-\mu_{1} x\right)\left(\mu_{2}-\mu_{3}\right)}{\left(\mu_{1}-\mu_{3}\right)\left(\mu_{1}-\mu_{2}\right)}\right)\right]+
\end{aligned}
$$




$$
\begin{aligned}
+\frac{1}{2} & {\left[\beta_{\mathrm{ч}}^{2}\left(\frac{y-\mu_{3} x}{\mu_{2}-\mu_{3}}\right)-\beta_{\mathrm{u}}^{2}\left(\frac{y-\mu_{1} x}{\mu_{1}-\mu_{2}}\right)-\beta_{\mathrm{ч}}^{2}\left(\frac{\left(y-\mu_{2} x\right)\left(\mu_{1}-\mu_{3}\right)}{\left(\mu_{1}-\mu_{2}\right)\left(\mu_{2}-\mu_{3}\right)}\right)\right]-} \\
& -\frac{1}{2}\left[\gamma_{\mathrm{u}}^{2}\left(\frac{\left(y-\mu_{3} x\right)\left(\mu_{1}-\mu_{2}\right)}{\left(\mu_{1}-\mu_{3}\right)\left(\mu_{2}-\mu_{3}\right)}\right)-\gamma_{\mathrm{u}}^{2}\left(\frac{y-\mu_{1} x}{\mu_{1}-\mu_{3}}\right)-\gamma_{\mathrm{u}}^{2}\left(\frac{y-\mu_{2} x}{\mu_{2}-\mu_{3}}\right)\right] .
\end{aligned}
$$

Ищем решение задачи $G$ в виде решения матричного уравнения $U=T V$ :

$$
\begin{gathered}
u^{1}(x, y)=\frac{\left(b_{22}+\frac{b_{12}}{a_{12}}\left(a_{1}-a_{22}\right)\right) b_{12}}{\delta_{1}} t_{1} v^{1}+\frac{\left(b_{22}+\frac{b_{12}}{a_{12}}\left(a_{2}-a_{22}\right)\right) b_{12}}{\delta_{1}} t_{2} v^{2}, \\
u^{2}(x, y)=t_{1} v^{1}+t_{2} v^{2} .
\end{gathered}
$$

Непосредственной подстановкой убеждаемся, что полученная вектор-функция $U(x, y)=\left(u^{1}(x, y), u^{2}(x, y)\right)$ удовлетворяет задаче $(4)$.

Проиллюстрируем вышеизложенное на примере системы

$$
A^{*} U_{x x x}+B^{*} U_{x x y}+C^{*} U_{x y y}+D^{*} U_{y y y}=0,
$$

у которой матрицы $A, D$ - нулевые, $B$ - единичная матрица второго порядка, $C=-Q$.

В плоскости независимых переменных $x, y \in \mathbb{R}$ рассмотрим одну систему гиперболических уравнений третьего порядка:

$$
U_{x x y}-Q U_{x y y}=0,
$$

где $U(x, y)=\left(u^{1}(x, y), u^{2}(x, y)\right)$ - вектор-функция, $Q-$ постоянная матрица вида

$$
Q=\left(\begin{array}{rr}
p & 1-p \\
1+p & -p
\end{array}\right), \quad p \in \mathbb{R}
$$

Существует матрица $T=\left(\begin{array}{cc}\frac{1}{\sqrt{2}} & \frac{p-1}{\sqrt{2\left(p^{2}+1\right)}} \\ \frac{1}{\sqrt{2}} & \frac{p+1}{\sqrt{2\left(p^{2}+1\right)}}\end{array}\right)$ такая, что $T^{-1} Q T=\Lambda_{Q}$, где $T^{-1}=\left(\begin{array}{cc}\frac{p+1}{\sqrt{2}} & -\frac{p-1}{\sqrt{2}} \\ -\sqrt{\frac{p^{2}+1}{2}} & \sqrt{\frac{p^{2}+1}{2}}\end{array}\right), \Lambda_{Q}=\left(\begin{array}{cc}1 & 0 \\ 0 & -1\end{array}\right)$.

Указанная система (5) имеет две кратные и две различные характеристики.

ЗАДАчА G1. Найти решение $U(x, y) \in C^{3}(\mathbb{R} \times \mathbb{R})$ системы уравнений (5), удовлетворяющее следующим условиям: $U(x, 0)=S \alpha(x), U(0, y)=S \beta(y)$, $\left\langle l_{1}, U(x,-x)\right\rangle=\gamma^{1}(x),\left\langle l_{2}, U(x, x)\right\rangle=\gamma^{2}(x)$, где $\alpha(x)=\left(\alpha^{1}(x), \alpha^{2}(x)\right), \beta(y)=$ $=\left(\beta^{1}(y), \beta^{2}(y)\right), \alpha^{i}(x), \beta^{i}(y), \gamma^{i}(x) \in C^{3}(\mathbb{R}), i=1,2,\langle a, b\rangle-$ скалярное произведение; $l_{1}=((p+1) / \sqrt{2},-(p-1) / \sqrt{2}), l_{2}=\left(-\sqrt{\left(p^{2}+1\right) / 2}, \sqrt{\left(p^{2}+1\right) / 2}\right)$; матрица $S-$ постоянная матрища вида $S=\left(\begin{array}{cc}\frac{1}{\sqrt{2}} & \frac{p-1}{\sqrt{2\left(p^{2}+1\right)}} \\ \frac{p+1}{\sqrt{2\left(p^{2}+1\right)}} & \frac{1}{\sqrt{2}}\end{array}\right)$.

Теорема 2. Если $\gamma_{\mathrm{H}}^{i}(x)=\alpha_{\mathrm{H}}^{i}(x)-\beta_{\mathrm{H}}^{i}(x), i=1,2$, то задача $G 1$ корректна по Адамару. 


\section{Доказ а тельст в о. Система (5) эквивалентна системе}

$$
V_{x x y}-\Lambda_{Q} V_{x y y}=0
$$

или

$$
\left\{\begin{array}{l}
v_{x x y}^{1}-v_{x y y}^{1}=0 \\
v_{x x x}^{2}+v_{x y y}^{2}=0
\end{array}\right.
$$

Вектор-функция $V(x, y)=\left(v^{1}(x, y), v^{2}(x, y)\right)$ - решение системы $(6)$, где

$$
\begin{aligned}
v^{1}(x, y)= & \alpha^{1}(x)+\beta^{1}(y)-\frac{1}{2} \alpha^{1}(0)-\frac{1}{2}\left[\alpha_{\mathrm{u}}^{1}(x)+\alpha_{\mathrm{u}}^{1}(y)-\alpha_{\mathrm{u}}^{1}(x+y)\right]- \\
& -\frac{1}{2}\left[\beta_{\mathrm{u}}^{1}(x)+\beta_{\mathrm{u}}^{1}(y)-\beta_{\mathrm{u}}^{1}(x+y)\right]+\frac{1}{2}\left[\gamma_{\mathrm{u}}^{1}(x)+\gamma_{\mathrm{u}}^{1}(y)-\gamma_{\mathrm{u}}^{1}(x+y)\right], \\
v^{2}(x, y)= & \alpha^{2}(x)+\beta^{2}(y)-\frac{1}{2} \alpha^{2}(0)-\frac{1}{2}\left[\alpha_{\mathrm{u}}^{2}(x)+\alpha_{\mathrm{u}}^{2}(y)-\alpha_{\mathrm{u}}^{2}(-x+y)\right]- \\
- & \frac{1}{2}\left[\beta_{\mathrm{u}}^{2}(x)+\beta_{\mathrm{u}}^{2}(y)-\beta_{\mathrm{u}}^{2}(-x+y)\right]+\frac{1}{2}\left[\gamma_{\mathrm{u}}^{2}(x)+\gamma_{\mathrm{u}}^{2}(y)-\gamma_{\mathrm{u}}^{2}(-x+y)\right] .
\end{aligned}
$$

Решение характеристической задачи $G 1$ для системы (5) ищем в виде решения матричного уравнения $U=T V$ :

$$
u^{1}(x, y)=\frac{1}{\sqrt{2}} v^{1}+\frac{p-1}{\sqrt{2\left(p^{2}+1\right)}} v^{2}, \quad u^{2}(x, y)=\frac{1}{\sqrt{2}} v^{1}+\frac{p+1}{\sqrt{2\left(p^{2}+1\right)}} v^{2}
$$

Полученная вектор-функция $U(x, y)=\left(u^{1}(x, y), u^{2}(x, y)\right)$, записанная в явном виде, является решением характеристической задачи $G 1$.

\section{БИБЛИОГРАФИЧЕСКИЙ СПИСОК}

1. А. В. Бицадзе, "К вопросу о постановке характеристической задачи для гиперболических систем второго порядка" // Докл. АН СССР, 1975. Т. 223, №6. С. 1289-1292. [A. V. Bitsadze, "On a question on the formulation of the characteristic problem for secondorder hyperbolic systems" // Dokl. Akad. Nauk SSSR, 1975. Vol. 223, no. 6. Pp. 1289-1292].

2. О. М. Джсхадзе, "Влияние младших членов на корректность постановки характеристических задач для гиперболических уравнений третьего порядка" // Maтем. заметки, 2003. Т. 74, № 4. С. 517-528; англ. пер.: O. M. Dzhokhadze, "Influence of Lower Terms on the Well-Posedness of Characteristics Problems for Third-Order Hyperbolic Equations" // Math. Notes, 2003. Vol. 74, no. 4. Pp. 491-501.

3. А. П. Солдатов, М. Х. Шхануков, "Краевые задачи с общим нелокальным условием Самарского А. А. для псевдопараболических уравнений высокого порядка" // Докл. АH CCCP, 1987. T. 297, № 3. С. 547-552; англ. пер.: A. P. Soldatov, M. Kh. Shkhanukov, "Boundary value problems with A. A. Samarskiy's general nonlocal condition for higherorder pseudoparabolic equations" // Soviet Math. Dokl., 1988. Vol. 36, no. 3. Pp. 507-511.

4. С. С. Харибегашвили, "О разрешимости одной характеристической задачи для вырождающихся систем второго порядка" // Диффер. уравнения, 1989. Т. 25, № 1. С. 154-162; англ. пер.: S. S. Kharibegashvili, "Solvability of a characteristic problem for degenerate second-order hyperbolic systems" // Differ. Equations, 1989. Vol. 25, no. 1. Pp. 123-131.

5. А. В. Бицадзе, Некоторые классы уравнений в частных производных. М.: Наука, 1981. 448 c. [A. V. Bitsadze, Some classes of partial differential equations. Moscow: Nauka, 1981. 448 pp.] 
6. Ю. О. Яковлева, "Одна характеристическая задача для дифференциального гиперболического уравнения третьего порядка общего вида с некратными характеристиками" // Вестн. Сам. гос. техн. ун-та. Сер. Физ.-мат. науки, 2012. №3(28). С. 180-183. [J. O. Yakovleva, "One characteristic problem for the general hyperbolic differential equation of the third order with nonmultiple characteristics" // Vestn. Samar. Gos. Tekhn. Univ. Ser. Fiz.-Mat. Nauki, 2012. no.3(28). Pp. 180-183].

Поступила в редакцию 16/XI/2012;

в окончательном варианте - 27/I/2013.

MSC: $35 \mathrm{L25}$

\section{THE CHARACTERISTIC PROBLEM FOR THE SYSTEM OF THE GENERAL HYPERBOLIC DIFFERENTIAL EQUATIONS OF THE THIRD ORDER WITH NONMULTIPLE CHARACTERISTICS}

\section{A. A. Andreev, J. O. Yakovleva}

Samara State Technical University, 244, Molodogvardeyskaya st., Samara, 443100, Russia.

E-mails: andre01071948@yandex.ru, julia.yakovleva@mail.ru

We consider the well-posed characteristic problem for the system of the general hyperbolic differential equations of the third order with nonmultiple characteristics. The solution of this problem is constructed in an explicit form. The example of the analogue of Goursat problem for a particular system of the hyperbolic differential equations of the third order is given.

Key words: system of the general hyperbolic differential equations, nonmultiple characteristics, characteristic problem, Hadamard's well-posedness.

Original article submitted 16/XI/2012; revision submitted $27 / \mathrm{I} / 2013$.

Aleksandr A. Andreev (Ph. D. (Phys. \& Math.)), Associate Professor, Dept. of Applied Mathematics \& Computer Science. Julia O. Yakovleva, Postgraduate Student, Dept. of Applied Mathematics \& Computer Science. 